\title{
Reporte del primer caso humano de infección parasitaria por Mammomonogamus laryngeus en Colombia
}

\author{
Jhon Carlos Castaño ${ }^{1}$, Fidel Ángel Núñez ${ }^{2}$, María Mercedes González ${ }^{1}$, Germán Téllez ${ }^{1}$, \\ María Isabel Giraldo ${ }^{1}$ \\ ${ }^{1}$ Grupo de Inmunología Molecular (GYMOL), Centro de Investigaciones Biomédicas, Universidad del \\ Quindío, Armenia, Colombia. \\ 2 Subdirección de Parasitología, Instituto de Medicina Tropical "Pedro Kourí", Ciudad de La Habana, Cuba. \\ Los parásitos del género Mammomonogamus afectan el tracto respiratorio de mamíferos \\ domésticos. La especie $M$. laryngeus es un nemátodo donde la hembra y el macho están \\ unidos en permanente cópula produciendo una forma distintiva de letra "Y" mayúscula. En el \\ mundo han sido reportados hasta el momento poco más de 100 casos de infección en humanos. \\ Este reporte describe el primer paciente infectado en Colombia (Armenia, Quindío), quien \\ presentó como sintomatología tos crónica productiva de dos meses de duración, con inmediata \\ y total recuperación clínica luego de la expulsión del parásito en el esputo durante un fuerte \\ acceso de tos. \\ Este reporte incluye la descripción morfológica del gusano adulto, así como un breve resumen \\ de la epidemiología y manifestaciones clínicas en humanos. También se presentan las \\ fotografías tomadas al parásito, donde se observan algunos de los detalles morfológicos \\ principales, que sirvieron para su identificación.
}

Palabras clave: parásitos/parasitología, insuficiencia respiratoria, informes de casos [Tipo de publicación], Colombia.

\section{First case report of Mammomonogamus (Syngamus) laryngeus human infection in Colombia}

Parasitic nematodes of the genus Mammomonogamus affect the respiratory tract of domestic mammals. The male and female of $M$. laryngeus remain in permanent copula so that the pair appears as a ' $Y$ '. To date, a few more than 100 cases of human infections by this parasite have been reported in the biomedical literature. This report describes the first infected patient in Colombia. He had a persistent and productive cough and after an episode of coughing a pair of worms were expelled in sputum with total clinical recovery.

Since there is scant clinical information about this parasite, this report includes a description of the adult worms, a summary of the epidemiology and the clinical manifestations in humans. Photographs are presented to facilitate future identification by morphological characteristics.

Key words: parasites/parasitology, respiratory insufficiency, case reports [Publication type], Colombia.

Mammomonogamus laryngeus (Syngamus laryngeus) (del griego syn, junto y gamos, matrimonio) es un parásito nemátodo que suele encontrarse en la laringe de mamíferos del trópico (1), especialmente en ovejas, bovinos, caprinos,

\section{Correspondencia:}

Jhon Carlos Castaño, carrera 15 con calle 12 N, Armenia, Quindío, Colombia.

Telefax: + (576) 7460168 .

gymol@uniquindio.edu.co

Recibido: 22/05/06; aceptado: 04/08/06 y gatos (2-4). La infección en humanos es rara, y hasta el momento se han reportado poco más de 100 casos, la mayoría en pacientes que visitaron Brasil y las islas del Caribe, de los cuales aproximadamente la mitad se originó en la isla de Martinica $(2,5,6)$. En Norteamérica se han reportado cinco casos, pero en todos ellos los pacientes refirieron antecedentes de haber visitado la zona del Caribe $(7,8)$. La syngamosis humana se ha reportado en las islas del Caribe y en Sur América, especialmente en Brasil; otros reportes 
provienen de Australia, Canadá, Estados Unidos, Reino Unido y Francia (3-5). En el continente asiático también existen casos reportados en Filipinas, Corea y Tailandia, aunque se piensa que en algunos de estos pacientes las infecciones habían sido adquiridas en China, Viet Nam y Malasia (9-11). En Colombia hasta ahora no se había reportado esta parasitosis en humanos y sólo se tiene referencia de un reporte de esta entidad en ganado bovino en el año de 1977 (12). Por esta razón cobra gran importancia la presentación de este caso que constituye, hasta donde sabemos, el primer informe de esta infección en humanos en Colombia.

\section{Presentación del caso}

Se trata de un paciente de sexo masculino, mestizo, de 18 años de edad, natural y procedente de Armenia, estudiante universitario y habitante en la zona urbana de la ciudad, quien consultó por haber eliminado "un pequeño gusano que se movía" después de un fuerte acceso de tos seca. EI paciente refirió antecedentes de tener un cuadro clínico de 60 días de evolución consistente en tos seca persistente con accesos, sin expectoración, ni hemoptisis y sensación de cuerpo extraño en laringe con dolor tipo ardor. Esta sintomatología se manejó con antitusivos, antihistamínicos (loratadina) y esteroides sistémicos, sin una mejoría clínica evidente. Después de 45 días, el paciente refirió haber eliminado un parásito vivo en el esputo durante un acceso de tos, después de lo cual desaparecieron los síntomas.

Como antecedentes epidemiológicos relevantes el paciente refirió visitas frecuentes al área rural (fincas de la zona rural) donde ha tenido contacto con ganado vacuno y contacto estrecho con un gato días antes del cuadro clínico.

La radiografía de tórax fue normal. A continuación se señalan los resultados de otros exámenes de laboratorio.

Cuadro hemático: hemoglobina, $16 \mathrm{mg} / \mathrm{dl}$; hematocrito, 48\%; leucocitos, 11000 ; neutrófilos, $73 \%$; leucocitos, $21 \%$; eosinófilos, $3 \%$; mastocitos, $2 \%$; basófilos, $1 \%$.

En cuanto a los resultados del estudio parasitológico de las heces, no se observaron formas parasitarias en el estudio del examen directo, con el método de frotis de Kato-Katz, ni por la técnica de concentración de formol éter modificada (Ritchie).

El examen de esputo fue negativo para huevos de M. laryngeus.

\section{Descripción del parásito}

Descripción macroscópica: se encontraron los dos parásitos en cópula, con forma de "Y" en el esputo del paciente, ambos de color rojo intenso, y en movimiento.

Descripción microscópica: usando el microscopio invertido se observó que el macho se encontraba copulando en el tercio anterior de la hembra. Es notable en la hembra la presencia en el extremo posterior de una terminación en punta, con una forma característica de flecha (figura 1). El ejemplar macho tenía un tamaño de 4,8 milímetros de largo y $0,37 \mathrm{~mm}$ de ancho, con un diámetro de la boca de $0,31 \mathrm{~mm}$, mientras que la hembra midió 9,6 milímetros de largo por 0,56 mm de ancho.

La cápsula bucal se observó desprovista de placas quitinosas o dientes externos, y se identificó por la forma característica de "copa" (figura 2). Todo esto nos llevó a la conclusión de que se trataba de una infección por M. laryngeus.

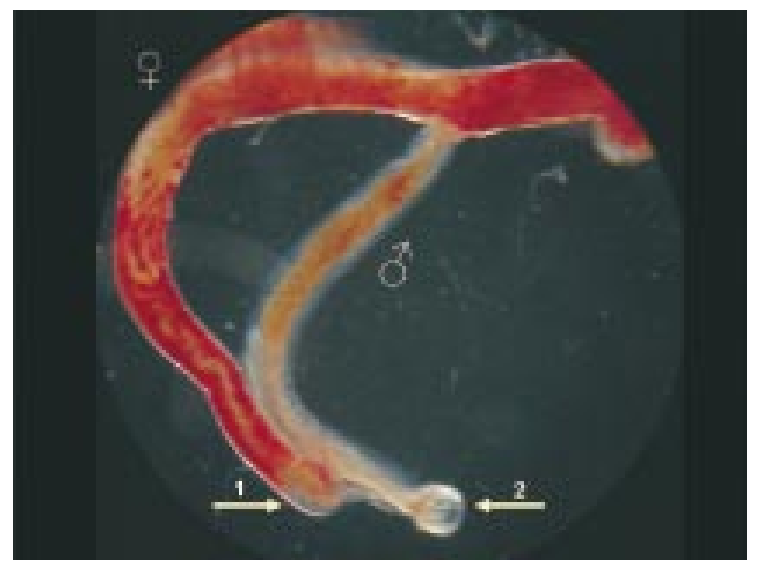

Figura 1. En la microfotografía se observan los dos ejemplares de Mammomonogamus laryngeus en cópula permanente. La flecha 1 señala la extremidad posterior de la hembra, mientras que la flecha 2 señala la cápsula bucal del macho. 


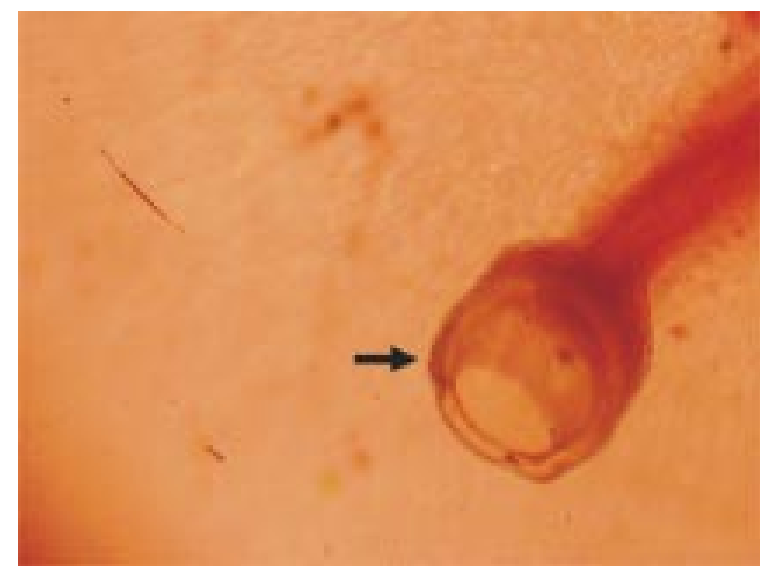

Figura 2. En la microfotografía de mayor aumento se observa la cápsula bucal en forma de copa del ejemplar macho de Mammomonogamus laryngeus.

\section{Discusión}

M. laryngeus es un nematodo de la superfamilia Strongyloidea, familia Syngamus, con diferentes géneros que parasitan a mamíferos domésticos (Mammomonogamus s, Ryzhikov), roedores (Rodentogamus, Sadovskaya) y aves (Syngamus, Smit). Sólo el género Mammomonogamus parasita a los humanos. Los parásitos son hematófagos, por lo que presentan una coloración que fluctúa entre el rosado y el rojo brillante.

La hembra y el macho se encuentran permanentemente en cópula y toman la forma característica de "Y" mayúscula, donde la hembra sería el brazo largo de la letra, y el macho el corto. Ambos sexos presentan una cápsula bucal de pared gruesa dirigida hacia adelante y armada en su base interna con ocho pequeños dientes internos que son aproximadamente iguales. En su unión con el esófago está provista de una pared muscular gruesa. Inmediatamente alrededor de la apertura oral hay un anillo cuticular grueso, y rodeando éste hay cuatro pares de labios en forma de pétalos, distribuidos en dos dorsales, dos ventrales, dos dorsolaterales y dos ventrolaterales. En la muesca que queda entre cada dos labios hay una papila pequeña. El macho es considerablemente más pequeño que la hembra y está permanentemente unido en cópula con ella. La bolsa copulatriz y sus radios de sostenimiento son característicos tanto del género como de la especie. Las espículas copulatrices son generalmente cortas y gruesas. La vulva está situada a corta distancia delante de la parte media del cuerpo de la hembra, la cual tiene el extremo posterior afilado $(8,10)$.

El macho mide de 2 a $6,3 \mathrm{~mm}$ de longitud y 0,360 a 0,380 mm de ancho con o sin espículas de 0,02 a $0,03 \mathrm{~mm}$ de largo, y la hembra tiene una longitud de 5 a 23,5 mm con 0,55 a 0,57 mm de ancho. La vulva está situada en la parte anterior del cuerpo. Los huevos no tienen opérculo, tienen una forma elipsoide $u$ ovoide, y miden alrededor de $45 \times 80$ $\mu \mathrm{m}(8,11)$. En nuestro caso, los tamaños encontrados para el parasito adulto estaban dentro del rango descrito, al igual que los detalles morfológicos.

El ciclo de vida de $M$. laryngeus no está bien dilucidado aún, sin embargo se piensa que puede ser similar al que ha sido descrito en las aves infectadas con Syngamus trachea. Los parásitos adultos normalmente se encuentran en la región laringotraqueal de los rumiantes. Los huevos son expulsados con la tos y deglutidos, y se les puede recuperar en esputo y más frecuentemente en la materia fecal. Una vez en el exterior del hospedero, los huevos se desarrollan hasta contener una larva infectante. Esta larva puede permanecer en el huevo o ser liberada en el ambiente. En los rumiantes, la infección se da por ingestión de alimentos y agua contaminada con la larva infectante. También la ingestión de un hospedero de transporte como las lombrices de tierra, caracoles o artrópodos puede ser otra fuente de infección. En el tracto digestivo se asume que la larva es liberada, cruza la pared intestinal hacia las venas mesentéricas, luego llega a las cámaras derechas del corazón y de allí pasa por la arteria pulmonar a los alvéolos pulmonares de donde migra al área laringotraqueal, lugar donde maduran y se aparean los gusanos adultos. Todo este ciclo dura aproximadamente tres semanas $(8,11,13)$.

La observación de que los huevos del parásito no se desarrollan a temperatura ambiente en la tráquea de los pacientes, y la valoración de que en muchos casos (sobre todo en viajeros procedentes de áreas endémicas), los primeros síntomas aparecen 6 a 11 días después de la contaminación ha permitido proponer otra hipótesis que explicaría un modo diferente de transmisión 
en los humanos. En estos casos, los pacientes podrían ser infectados por parásitos adultos, que emigran de la faringe a la laringe y la tráquea por razones desconocidas, después de la ingestión de alimentos o agua contaminados por los hospederos de transporte (9).

Las manifestaciones clínicas de esta parasitosis se producen como consecuencia de la invasión del tracto respiratorio, principalmente de la mucosa del tracto respiratorio superior, es decir de laringe, tráquea o bronquios, y consisten fundamentalmente en tos crónica, que puede ser con expectoración o, más frecuentemente, no productiva, síntomas asmatiformes, molestia laríngea y puede llegar a producir hemoptisis. Sólo existe un caso inusual, recientemente publicado, en el que se detectaron parásitos adultos en el duodeno de un paciente de origen tailandés (11).

El diagnóstico se realiza usualmente mediante la observacióne directa del parásito en la faringe, en la laringe por laringoscopia, en los bronquios por broncoscopia, o por la identificación del parásito cuando es expulsado espontáneamente durante un acceso de tos. En todos estos casos se pueden encontrar los parásitos con la característica forma de " $Y$ ".

En ocasiones se pueden observar los huevos en las heces por ingestión del esputo, como ocurre por ejemplo en las infecciones pulmonares con Paragonimus. Los huevos se pueden observar también en la secreción bronquial; presentan metámeras por ser huevos de nemátodos del orden Strongylida, lo que pudiera traer como consecuencia la confusión con huevos de ancylostomídeos (Necator americanus o Ancylostoma duodenale).

En los pocos casos humanos publicados se ha producido mejoría en el paciente tras la extracción del parásito por laringoscopia, traqueoscopia o por la eliminación espontánea del parásito al toser, como ocurrió con este paciente. Entre los fármacos que se han usado para el tratamiento de la syngamosis se han descrito esquemas con tiabendazol $(8,14)$, mebendazol $(8,15)$ y albendazol $(3,9)$.

El paciente recibió tratamiento con $200 \mu \mathrm{g} / \mathrm{kg}$ de ivermectina en dosis única (16). Posteriormente se le realizó el seguimiento mediante dos controles semanales en los que resultó asintomático.

El joven no refirió ningún antecedente en cuanto a viajes a zonas endémicas de otros países donde se haya reportado esta parasitosis. El único antecedente epidemiológico positivo fue el contacto con gatos domésticos y viajes recreativos frecuentes a fincas de la región donde existe ganado bovino, situación que podría representar la posible endemicidad de esta parasitosis en la región. A pesar de que en otros países vecinos como en Brasil se han reportado más de 20 casos de infección humana, hasta donde sabemos éste es el primer caso de infección humana por M. laryngeus publicado en Colombia. Sólo se tiene referencia de un reporte de esta entidad en ganado bovino en el año de 1977 en el departamento del Valle del Cauca, colindante con el departamento del Quindío (12).

La presentación de este primer caso colombiano constituye una alerta a los clínicos y otros médicos relacionados con la atención a pacientes para que estén pendientes ante la presencia de manifestaciones clínicas de tos seca persistente y sensación de cuerpo extraño en la garganta, además de cuadros asmatiformes en los habitantes del departamento del Quindío, y en otras regiones del país donde esta infección pudiera ser una enzootia con un alto riesgo de transmisión zoonótica.

\section{Referencias}

1. Anderson RC, Chabaud AG, Willmott S. CIH keys to the nematode parasites of vertebrates, no. 7. Keys to the genera of the superfamily Strongyloidea. Farrham Royal, Bucks, England: Commonwealth Agricultural Bureau; 1980.

2. Beaver PC, Jung RC, Wayne E. Clinical Parasitology. Philadelphia: Lea and Febiger; 1984.

3. Da Costa JC, Delgado ML, Vieira P, Afonso A, Conde B, Cross JH. Syngamoniasis in tourist. Emerg Infect Dis 2005;11:1976-7.

4. Freitas AL, De Carli G, Blankenhein $\mathbf{M H}$. Mammomonogamus (Syngamus) laryngeus infection: a new Brazilian human case. Rev Inst Med Trop São Paulo 1995;37:177-9.

5. Cunnac M, Magnaval JF, Cayarci D, Leophonte P. A propos de 3 cas de syngamose humaine en Guadeloupe. Rev Pneumol Clin 1988;44:40-2.

6. Mornex JF, Magdeleine J, De Thore J. La syngamose humaine (Mammomonogamus nasicola) cause de toux 
chronique en Martinique. 37 observations recentes. Nouv Presse Med 1980;9:3628.

7. Pontes PA, Gadelha ME, Gregorio LC. Pathologic quiz case 2. Laryngeal syngamosis. Arch Otolaryngol Head Neck Surg 1993;119:573-4.

8. Nosanchuk JS, Wade SE, Landolf M. Case report of and description of parasite in Mammomonogamus laryngeus (human syngamosis) infection. J Clin Microbiol 1995;33:998-1000.

9. Kim HY, Lee SM, Joo JE, Na MJ, Ahh MH, Min DY. Human syngamosis: the first case in Korea. Thorax 1998 53:717-8.

10. Limawongpranee S, Samanthai S, Yoolek A. Human Mammomonogamus laryngeus infection (Syngamosis): The second case report in Thailand. Siriraj Hosp Gaz 2004;56:82-6.

11. Eamsobhana P, Mongkolporn T, Punthuprapasa P, Yoolek A. Mammomonogamus roundworm (Nematoda: Syngamidae) recovered from the duodenum of a
Thai patient: a first and unusual case originating in Thailand. Trans R Soc Trop Med Hyg 2006;100:387-91.

12. Guzman VH, Adams LG, Galvin TJ. Bovine laryngeus verminosis due to Mammonogamus laryngeus in Colombia. Revista ICA 1977;11:81-8.

13. Acha PN, Szyfres B. Zoonoses and communicable diseases common to animals and man. 2nd ed. Washington D.C: Pan American Health Organization; 1987.

14. Leers WD, Sarin MK, Arthurs K. Syngamosis, an unusual cause of asthma: the first reported case in Canada. Can Med Assoc J 1985;132:269-70.

15. Pipitgool V, Chaisiri K, Visetsupakarn P, Srigan V, Maleewong W. Mammonogamus (Syngamus) laryngeus infection: a first case report in Thailand. Southeast Asian J Trop Med Public Health 1992; 23: 336-7.

16. Geary TG. Ivermectin 20 years on: maturation of a wonder drug. Trends Parasitol 2005;21:530-2. 